\title{
Higher Order Solitary Wave Solutions of the Standard KdV Equations
}

\section{Clovis Taki Djeumen Tchaho1,2*, Hugues Martial Omanda1,3, Gaston N'tchayi Mbourou4, Jean Roger Bogning5, Timoléon Crépin Kofanée,7}

\author{
${ }^{1}$ African Centre for Advanced Studies, Yaoundé, Cameroon \\ ${ }^{2}$ Lycée Technique Fulbert Bongotha, Moanda, Gabon \\ ${ }^{3}$ Laboratoire Pluridisciplinaire des Sciences, Ecole Normale Supérieure, Libreville, Gabon \\ ${ }^{4}$ Laboratoire de Mécanique des Matériaux, Ecole Polytechnique, Université des Sciences et Techniques de Masuku, Franceville, \\ Gabon \\ ${ }^{5}$ Department of Physics, Higher Teacher Training College, University of Bamenda, Bamenda, Cameroon \\ ${ }^{6}$ Department of Physics, Faculty of Science, University of Yaoundé I, Yaoundé, Cameroon \\ ${ }^{7}$ Centre d'Excellence Africain en Technologie de l'Information et de la Télécommunication, University of Yaoundé I, Yaoundé, \\ Cameroon \\ Email: ^djeumenclovis@gmail.com,^djeumentchaho@acas-yde.org
}

How to cite this paper: Tchaho, C.T.D. Omanda, H.M., Mbourou, G.N., Bogning, J.R. and Kofané, T.C. (2021) Higher Order Solitary Wave Solutions of the Standard KdV Equations. Open Journal of Applied Sciences, 11, 103-125.

https://doi.org/10.4236/ojapps.2021.111008

Received: December 13, 2020

Accepted: January 26, 2021

Published: January 29, 2021

Copyright $\odot 2021$ by author(s) and Scientific Research Publishing Inc. This work is licensed under the Creative Commons Attribution International License (CC BY 4.0).

http://creativecommons.org/licenses/by/4.0/

\begin{abstract}
Considered under their standard form, the fifth-order KdV equations are a sort of reading table on which new prototypes of higher order solitary waves residing there, have been uncovered and revealed to broad daylight. The mathematical tool that made it possible to explore and analyze this equation is the Bogning-Djeumen Tchaho-Kofané method extended to the new implicit Bogning' functions. The analytical form of the solutions chosen in this manuscript is particular in the sense that it contains within its bosom, a package of solitary waves made up of three solitons, especially, the bright type soliton, the hybrid soliton and the dark type soliton which we estimate capable in their interactions of generating new hybrid or multi-form solitons. Existence conditions of the obtained solitons have been determined. It emerges that, these existence conditions of the chosen ansatz could open the way to other new varieties of fifth-order $\mathrm{KdV}$ equations including to which it will be one of the solutions. Some of the obtained solitons are exact solutions. Intense numerical simulations highlighted numerical stability and confirmed the hybrid character of the obtained solutions. These results will help to model new nonlinear wave phenomena, in plasma media and in fluid dynamics, especially, on the shallow water surface.
\end{abstract}

\section{Keywords}

Standard KdV Equations, Bogning-Djeumen Tchaho-Kofané Method, Higher 
Order Solitary Wave, Multi-Form Solitons, New Implicit Bogning' Functions

\section{Introduction}

Many natural phenomena regularly occur in the universe. These phenomena can in certain cases be destructive for the environment in which they take place. For example, the cases of the propagation of the nonlinear excitations in fluid dynamics which sometimes, are manifested by tsunamis, hurricanes, tidal bore, and so on, and which very often are devastating when surge is across on the continent without warning. These different phenomena as presented in this case (among so many others) constitute real and permanent threats to our existence on the earth planet. It is following this observation that, a great number of researchers in particular, physicists and mathematicians, deploy themselves in everyday life in order to design and make available mathematical models [1] [2] [3] [4] [5] in an attempt to analyze, understand and explain these phenomena that can be observed in various fields such as nonlinear optics, mechanics, biology, and so on. Most of these models are nonlinear partial differential equations (NPDEs). From all these equations, we are interested, in this manuscript, in the one whose history throughout the last two centuries is intimately linked from the outset to the remarkable scientific discovery made in August 1834 [6] by John Scott Russell and who shows up under the form

$$
w_{t}+\alpha w^{2} w_{x}+\beta w_{x} w_{x x}+\gamma w w_{x x x}+w_{x x x x x}=0 .
$$

Equation (1) is the standard form of the well-known fifth-order KdV (fKdV) equations [7]-[13] where $\alpha, \beta, \gamma$ are real and nonzero arbitrary parameters, $w(x, t)$ is a sufficiently differentiable function, and, $x, t$ are both independent spatial and temporal coordinates respectively. $w_{x x x}$ and $w_{x x x x}$ are the two dispersive terms involved in this equation. Equation (1) is susceptible to a radical change in its characteristics due to the arbitrary and varied characters of the values that the parameters $\alpha, \beta, \gamma$ can take. This changing and arbitrary character of the values that these different parameters can take gives rise to a variability of the fKdV equations [7] which are of particular interest in the literature and of which the best known are the Lax equation, the Ito equation, the Caudrey-Dodd-Gibbon (CDG) equation, the Sawada-Kotera (SK) equation and the Kaup-Kuperschmidt (KP) equation. Basically, the KdV equation models the propagation of weakly nonlinear dispersive waves in various fields such as plasma physics [14], waves of small amplitudes and long wavelengths on the surface of shallow waters (incompressible irrotational inviscid fluid) [15] [16] [17], the laser beam propagation [18], and so on. It is fundamental to point out here that the NPDEs admit different types of solutions, the most robust of which are solitary waves (solitons). However, one of the major difficulties remains the determination of the exact, approximate or forced solutions of these equations. In re- 
cent decades, numerous works have proposed various methods [19]-[26] of resolution in order to understand and explain phenomena which occur in systems whose dynamics are governed by these NLPDEs. Despite all this progress, a lot of work remains to be done because many other phenomena at the origin of the new predictable or unpredictable behaviors of these different systems still have to be detected, understood and explained in order to guarantee all of humanity a future safely.

So, in this manuscript, One tracks down, using the Bogning-Djeumen Tchaho-Kofané method (BDKm) extended to the new implicit Bogning' (iB) functions, new prototypes of solitary waves of the standard $\mathrm{KdV}$ equation while revealing the hybrid character of these waves. Section 2 will be devoted to the brief description of the BDKm including new implicit Bogning' functions (iB-functions) while, Section 3 will focus on finding the analytical solutions coupled with an intense numerical simulation. Section 4 will provide the gist of discussions. A conclusion followed by a perspective will complete this work in Section 5 .

\section{The BDKm Theory}

This section takes care of highlighting all the mechanisms necessary for the implementation of the BDKm including iB-functions which will allow in section 3, to unearth new prototypes of the solitary wave solutions of Equation (1).

\section{1. iB-Functions}

These iB-functions [23] [27] [28] [29] have been highlighted thanks to the multiple research works [22] [23] [28]-[44] produced for more than a decade. It is during the repeated constructions of solitary wave solutions of certain types of equations in wave mechanics presenting dispersion terms coupled with non-linear terms (which can be of different orders) via the BDKm, that the fascinating properties of these functions were detected by Bogning Jean Roger. These iB-functions are noted [23] [27] [28] [29]

$$
J_{n, m}\left(\sum_{i=0}^{p} \alpha_{i} x_{i}\right)=\frac{\sinh ^{m}\left(\sum_{i=0}^{p} \alpha_{i} x_{i}\right)}{\cosh ^{n}\left(\sum_{i=0}^{p} \alpha_{i} x_{i}\right)}=\sinh ^{m}\left(\sum_{i=0}^{p} \alpha_{i} x_{i}\right) \operatorname{sech}^{n}\left(\sum_{i=0}^{p} \alpha_{i} x_{i}\right) .
$$

The left member is the implicit form and the right member is the explicit form of the iB-functions, where $\alpha_{i},(i=0 ; 1 ; 2 ; \cdots ; p)$ are the parameters associated to the independent variables $x_{i},(i=0 ; 1 ; 2 ; \cdots ; p), m$ is the power of the numerator, $n$ that of the denominator. In one dimensional, according to the choice of the parameter $\alpha_{i}$, Equation (2), is reduces under the form

$$
J_{n, m}(\alpha x)=\frac{\sinh ^{m}(\alpha x)}{\cosh ^{n}(\alpha x)}=\sinh ^{m}(\alpha x) \operatorname{sech}^{n}(\alpha x),
$$

where $m$ and $n$ are keeping the same characteristics as in Equation (2), $\alpha$ is a constant associated to the independent variable $x$. We associate here some of the 
fundamental properties of these functions, whose a large majority of which will be useful in the rest of this manuscript under the respective forms

$$
\begin{gathered}
\frac{\mathrm{d}^{p} J_{n, m}}{\mathrm{~d} x^{p}}=m \alpha \frac{\mathrm{d}^{p-1} J_{n-1, m-1}}{\mathrm{~d} x^{p-1}}-n \alpha \frac{\mathrm{d}^{p-1} J_{n+1, m+1}}{\mathrm{~d} x^{p-1}}, \\
J_{n, m}^{p}=J_{n p, m p}, \\
J_{n, n}^{p}=J_{p, p}^{n}, \\
J_{-n, 0}(x+y)=\sum_{k=0}^{n} C_{n}^{k} J_{-n+k, k}(x) J_{-n+k, k}(y), \\
J_{0, n}(x+y)=\sum_{k=0}^{n} C_{n}^{k} J_{-k, n-k}(x) J_{-n+k, k}(y), \\
J_{2 n+1,2 n+1}=J_{1,1}\left(1-J_{2,0}\right)^{n}, \\
\mathrm{e}^{n x}=\sum_{k=0}^{n} C_{n}^{k} J_{-n+k, k}(x),
\end{gathered}
$$

and

$$
\mathrm{e}^{-n x}=\sum_{k=0}^{n}(-1)^{k} C_{n}^{k} J_{-n+k, k}(x) .
$$

It is important to note here that this function in its trigonometric form is written as

$$
J_{n, m}(i x)=(i)^{m} \frac{\sin ^{m}(x)}{\cos ^{n}(x)}
$$

although we should not use it for this work, but for the knowledge of the reader. For a better understanding of the properties of these functions, it is needful to refer to [21] [25] [26] [27] where they are widely explained.

\subsection{Implementation of the BDKm}

The BDKm which has been proposed by three Cameroonian researchers finds its implementation field in nonlinear physics, wave mechanics, mathematics physics, and others. It is better suited for solving certain types of NPDEs of the form [22] [23] [28]-[44]

$$
X\left(\Phi, \Phi_{t}, \Phi_{x}, \Phi_{t t}, \Phi_{x x}, \Phi_{t t t}, \Phi_{x x x}, \cdots,|\Phi|^{2},\left(\Phi|\Phi|^{2}\right)_{t}, \cdots\right)=0,
$$

where $\Phi(x, t)$ is an unknown function to be determined, $X$ is some function of $\Phi$ and its derivatives with respect to $x$ and $X$ includes the highest order derivatives and the nonlinear terms. Generally, the solution sought is of the form $\lambda_{i j} J_{i, j}\left(\sum_{k=0}^{p} \alpha_{k} x_{k}\right)$. As in the logic of a only variable, we can set the change of variable $\xi=\sum_{k=0}^{p} \alpha_{k} x_{k}$. But in the case where we have a function of $x$ and $t$, $\Phi(x, t)$, we can pose the change of variable $\xi=x-v t$. Thus, $\Phi(x, t)$ becomes $\Phi(\xi)$ where $v$ is the speed of the wave and Equation (13) becomes in these conditions 


$$
X_{O D E}\left(\Omega, \Omega^{\prime}, \Omega^{\prime \prime}, \cdots, \Omega^{\prime}|\Omega|^{2}, \cdots\right)=0 .
$$

Equation (14) is an ordinary differential equation (ODE), where $\Omega^{\prime}, \Omega^{\prime}$ represent respectively the first and second derivatives of the envelope $\Omega$ with respect to $\xi$. According to Equation (3), the solution we are trying to construct can be expressed as

$$
\Omega(\xi)=\sum_{i j} \mu_{i j} J_{j, i}(\eta \xi)
$$

where $\eta$ is a real constant and $\mu_{i j}$ are the unknown constants to be determined. So, the combination of Equations (14) and (15) gives the main equation

$\sum_{i j n} A_{n}\left(\mu_{i j}, \eta, v\right) J_{n, 0}(\eta \xi)+\sum_{i j m} B_{m}\left(\mu_{i j}, \eta, v\right) J_{m, 1}(\eta \xi)+\sum_{i j k} C_{k}\left(\mu_{i j}, \eta, v\right) J_{-k, 0}(\eta \xi)$

$+\sum_{i j l} D_{l}\left(\mu_{i j}, \eta, v\right) J_{-l, 1}(\eta \xi)+\sum_{i j} E\left(\mu_{i j}, \eta, v\right) J_{0,0}(\eta \xi)=0$,

where $i, j, k, l$ are positive natural integers and $n, m$ the real numbers [23] [27] [28] [29]. It can be noted here that Equation (16) is the one from which all the possible analyzes result. The identification of coefficients

$A_{n}\left(\mu_{i j}, \eta, v\right), B_{m}\left(\mu_{i j}, \eta, v\right), C_{k}\left(\mu_{i j}, \eta, v\right), D_{l}\left(\mu_{i j}, \eta, v\right), E\left(\mu_{i j}, \eta, v\right)$ at zero makes it possible to obtain the ranges of equations whose the resolutions could allow to obtain the expressions of the unknown coefficients $\mu_{i j}$. It is important to point out here that, the resolution of this series of equations often leads to exact solutions [30] [38] for certain models and according to the form of the considered ansatz while, for other models and according to the form of the chosen ansatz, it (resolution) leads to approximate or forced solutions. In the case of approximate or forced solutions, the priority in the order of resolution is given to those from the highest clues of $J_{n, 0}(\eta \xi)$, then to those of the highest clues of $J_{m, 1}(\eta \xi)$. But, otherwise we go to those from the coefficients of lowest clues of $J_{-k, 0}(\eta \xi)$ and $J_{-l, 1}(\eta \xi)$. Here, the priority makes reference to the series that permits to obtain good results or merely that tends more to the sought exact solution. Very often, the series of equations obtained by identifying at zero the coefficient of $J_{n, 0}(\eta \xi)$ gives satisfaction. On the opposite, the last series of equations given by the coefficient of $J_{0,0}(\eta \xi)$ is not very important because it is considered rather like a confused domain for the obtainable good solutions. So, this resolution permits to obtain the possible expressions of the coefficients $\mu_{i j}$ of Equation (15) as a function of the parameters $\eta, v$, and those supplied by Equation (13), and then of the constraints which may result therefrom. Thus, the ansatz given in Equation (15) can be supported by Equation (13) as a solution. Recently, this method was used in [36] [37] and in [42] [43] [44] to construct hybrid solitary waves for the generalized Kuramoto-Sivashinsky equation, Multi-form solitary wave solutions of the KdV-Burgers-Kuaramoto equation and solitary wave solutions which propagate through transmission media such as electrical lines, respectively. Now, we apply it again to the standard form of the well-known fifth-order KDV equation [7]. 


\section{Results}

This part of the work is grouped into two subsections. The first subsection deals with the construction of the analytical hybrid solitary wave solutions of Equation (1). The second sub-section, for its part, is working on an intense numerical simulation in order to reassure itself of the stability of the obtained solutions with a view to a probable future application and for a possible confirmation of the hybrid characters (planned when choosing of the ansatz given by Equation (18) below) of these obtained solutions.

\subsection{Analytical Higher Order Solitary Wave Solutions}

By considering the change of variable $w(x, t)=\psi(\xi)$ where $\xi=x-v t$ and $v$ the wave speed, Equation (1) becomes

$$
v \psi_{\xi}-\alpha \psi^{2} \psi_{\xi}-\beta \psi_{\xi} \psi_{2 \xi}-\gamma \psi_{\xi} \psi_{3 \xi}-\psi_{5 \xi}=0
$$

where $\psi_{s \xi}=\frac{\partial^{s} \psi}{\partial \xi^{s}}$. We are looking for the solutions under the form

$$
\psi(\xi)=a J_{1,0}(\eta \xi)+b J_{1,0}(\eta \xi) J_{1,1}(\eta \xi)+c J_{2,2}(\eta \xi),
$$

where $a, b$ and $c$ are real constants to be determined later, $\eta$, the inverse of the width of each component of Equation (18). It should be noted here that Equation (18) has a peculiarity in the sense that it is a collection of solitons taken individually in the two main families of existing solitary waves, namely the bright family and the dark family to make it a package [23] [27] [28] [29]. To be more precise, the first term of Equation (18) is a bright represented by the sech function, the second term is a hybrid soliton obtained by performing the product of a bright by a dark represented by $\operatorname{sech} \times \tanh$ and the third term is a dark represented by $\tanh ^{2}$. One just has to represent each of the terms to realize it. In this context, we believe that this mixture can be at the origin of interactions (or competitions) between these different components and generate hybrid structures, this, in relation to the values taken by the coefficients $a, b, c$ and the parameters $\eta, v$ of the wave, as well as those taken by the parameters $\alpha, \beta, \gamma$ of the considered system. Following all that has just been said, we continue with our investigations by inserting Equation (18) into Equation (17) to produce of ranges main equation and contracted under the form

$$
\sum_{u} K_{u}(a, b, c, \alpha, \beta, \gamma, \eta, v) J_{u, 0}(\eta \xi)+\sum_{v} Z_{v}(a, b, c, \alpha, \beta, \gamma, \eta, v) J_{v, 1}(\eta \xi)=0,
$$

where $u \in\{1 ; 2 ; 3 ; 4 ; 5 ; 6 ; 7\} ; v \in\{2 ; 3 ; 4 ; 5 ; 6 ; 7\}$. So, Equation (19) has delivered in its formulation, two ranges of equations in the terms of $J_{u, 0}(\alpha \xi)$, and $J_{v, 1}(\alpha \xi)$, thus constituting the most important ranges according to the implementation of the above BDKm. In this context, priority in solving is given to the equations from the first range that is to say, the equations from the coefficients of the terms in $J_{u, 0}(\alpha \xi), u \in\{1 ; 2 ; 3 ; 4 ; 5 ; 6 ; 7\}$ due to the complexity of the equations of the second range. Thus, by identifying at zero the different coefficients $K_{u}, u \in\{1 ; 2 ; 3 ; 4 ; 5 ; 6 ; 7\}$ of the terms in 
$J_{u, 0}(\alpha \xi), u \in\{1 ; 2 ; 3 ; 4 ; 5 ; 6 ; 7\}$ and the different coefficients

$Z_{v}, v \in\{2 ; 3 ; 4 ; 5 ; 6 ; 7\}$ of the terms in $J_{v, 1}(\alpha \xi), v \in\{2 ; 3 ; 4 ; 5 ; 6 ; 7\}$, we obtain the both series of algebraic equations of unknowns $a, b$ and $c$ as being the term in $J_{7,0}(\alpha \xi)$,

$$
\left[2 \eta \alpha b^{2}-6 \eta \alpha c^{2}-\left(24 \eta^{3} \beta+48 \eta^{3} \gamma\right) c-720 \eta^{5}\right] b=0,
$$

the term in $J_{6,0}(\alpha \xi)$,

$$
\left(10 \eta^{3} \beta+30 \eta^{3} \gamma+10 \eta \alpha c\right) a b=0
$$

the term in $J_{5,0}(\alpha \xi)$,

$$
\left[13 \eta \alpha c^{2}-4 \eta \alpha a^{2}-3 \eta \alpha b^{2}+\left(28 \eta^{3} \beta+76 \eta^{3} \gamma\right) c+840 \eta^{5}\right] b=0,
$$

the term in $J_{4,0}(\alpha \xi)$,

$$
\left(-14 \eta \alpha c-11 \eta^{3} \beta-27 \eta^{3} \gamma\right) a b=0
$$

the term in $J_{3,0}(\alpha \xi)$,

$$
\left[3 \eta \alpha a^{2}+\eta \alpha b^{2}-8 \eta \alpha c^{2}-\left(6 \eta^{3} \beta+29 \eta^{3} \gamma\right) c+2 \eta v-182 \eta^{5}\right] b=0,
$$

the term in $J_{2,0}(\alpha \xi)$,

$$
\left(4 \eta \alpha c+2 \eta^{3} \beta+2 \eta^{3} \gamma\right) a b=0,
$$

the term in $J_{1,0}(\alpha \xi)$,

$$
\left(\eta \alpha c^{2}+\eta^{3} \gamma c+\eta^{5}-\eta v\right) b=0
$$

the term in $J_{7,1}(\alpha \xi)$,

$$
\left(6 \eta^{3} \beta+24 \eta^{3} \gamma\right) b^{2}-2 \eta \alpha c^{3}+6 \eta \alpha b^{2} c-\left(12 \eta^{3} \beta+24 \eta^{3} \gamma\right) c^{2}-720 \eta^{5} c=0,
$$

the term in $J_{6,1}(\alpha \xi)$,

$$
\left[5 \eta \alpha c^{2}-5 \eta \alpha b^{2}+\left(10 \eta^{3} \beta+30 \eta^{3} \gamma\right) c+59 \eta^{5}\right] a=0,
$$

the term in $J_{5,1}(\alpha \xi)$,

$$
\begin{aligned}
& 4 \eta \alpha c^{3}+\left(8 \eta^{3} \beta+32 \eta^{3} \gamma\right) c^{2}-\left(2 \eta^{3} \beta+6 \eta^{3} \gamma\right) a^{2} \\
& -\left(8 \eta^{3} \beta+20 \eta^{3} \gamma\right) b^{2}-4 \eta \alpha a^{2} c-8 \eta \alpha b^{2} c+480 \eta^{5} c=0,
\end{aligned}
$$

the term in $J_{4,1}(\alpha \xi)$,

$$
\left[\eta \alpha a^{2}+3 \eta \alpha b^{2}-6 \eta \alpha c^{2}-\left(6 \eta^{3} \beta+15 \eta^{3} \gamma\right) c-60 \eta^{5}\right] a=0
$$

the term in $J_{3,1}(\alpha \xi)$,

$$
\begin{aligned}
& \left(\eta^{3} \gamma+2 \eta^{3} \beta\right) a^{2}+\left(\eta^{3} \gamma+\eta^{3} \beta\right) b^{2}-2 \eta \alpha c^{3}-8 \eta^{3} \gamma c^{2} \\
& +2 \eta \alpha a^{2} c+2 \eta \alpha b^{2} c+\left(2 \eta v-32 \eta^{5}\right) c=0
\end{aligned}
$$

the term in $J_{2,1}(\alpha \xi)$,

$$
\left(\eta \alpha c^{2}+\eta^{3} \gamma c+\eta^{5}-v \eta\right) a=0 .
$$

At look to the structuring of the equations unveiled by this range, one can eas- 
ily make the choices of the different families of the solutions to be constructed. Thus, in order to obtain non-trivial solutions, we will distinguish four cases from families of solutions, notably the case $a \neq 0, b \neq 0$; the case $a=0, b \neq 0$; the case $b=0, a \neq 0$ and the case $a=0, b=0$. Thus, these investigations plunge us straight into what is essential in Subsection 3.1.

\subsubsection{First Family of Solutions: Case: $a \neq 0 ; b \neq 0$}

From Equations (21), (23) and (25), one obtains, respectively

$$
\begin{gathered}
c=-\frac{\eta^{2} \beta+3 \eta^{2} \gamma}{\alpha}, \\
c=-\frac{11 \eta^{2} \beta+27 \eta^{2} \gamma}{14 \alpha}
\end{gathered}
$$

and

$$
c=-\frac{\eta^{2} \beta+\eta^{2} \gamma}{2 \alpha},
$$

with $\alpha \neq 0$. By setting the equality $c=c$ between Equations (33) and (35) on the one hand, then, between Equations (34) and (35) on the other hand, it comes the constraint verified by the parameters $\gamma$ and $\beta$ below

$$
\beta=-5 \gamma
$$

The insertion of Equation (36) into one of Equations (33), (34) and (35) gives

$$
c=2 \eta^{2} \frac{\gamma}{\alpha}
$$

It emerges from Equation (37) that, the coefficient $c$ is a linear function of parameter $\gamma$ or that $c$ is a hyperbolic function of parameter $\alpha(\alpha \neq 0)$ or that $c$ is a parabolic function of the inverse of the width at half height $\eta$ of the solitons, that one is searches to constructing according to the fact that the choice of the variable be worn on one of the parameters $\gamma, \alpha$ or $\eta$. These observations sufficiently show what will be the importance of the impact of the variations of the coefficient $c$ on the formation of the wave structures that we will obtain. By continuing, the insertion of Equation (37) into Equation (20) and Equation (26) gives, successively

$$
\begin{gathered}
b^{2}=-60 \eta^{4} \frac{\gamma^{2}}{\alpha^{2}}+360 \eta^{4} \frac{1}{\alpha}, \\
b= \pm \frac{\eta^{2}}{\alpha} \sqrt{360 \alpha-60 \gamma^{2}} ; \alpha \succ \frac{1}{6} \gamma^{2}
\end{gathered}
$$

and

$$
v=6 \eta^{4} \frac{\gamma}{\alpha}+\eta^{4}
$$

Equation (40) reveals that the speed $v$ has almost the same variations as in the case of Equation (37) with respect to the parameters $\gamma, \beta$ and $\eta$. Taking into account Equations (37) and (38) in the Equation (22) gives, respectively 


$$
a^{2}=26 \eta^{4} \frac{\gamma^{2}}{\alpha^{2}}-60 \eta^{4} \frac{1}{\alpha}
$$

and

$$
a= \pm \frac{\eta^{2}}{\alpha} \sqrt{26 \gamma^{2}-60 \alpha} ; \alpha \prec \frac{13}{30} \gamma^{2}
$$

It is important to emphasize here that, the two conditions which validate Equations (39) and (42) impose on to take $\alpha \in] \frac{1}{6} \gamma^{2} ; \frac{13}{30} \gamma^{2}[$ and $\alpha \neq 0$. And also, Equations (36), (37), (38), (40) and (41) verify Equation (24). We thus obtain the first family of solutions under the form

$$
\begin{aligned}
\psi(\xi)= & \pm \frac{\eta^{2}}{\alpha} \sqrt{26 \gamma^{2}-60 \alpha} J_{1,0}(\eta \xi) \\
& \pm \frac{\eta^{2}}{\alpha} \sqrt{360 \alpha-60 \gamma^{2}} J_{1,0}(\eta \xi) J_{1,1}(\eta \xi)+2 \eta^{2} \frac{\gamma}{\alpha} J_{2,2}(\eta \xi),
\end{aligned}
$$

with $\alpha \in] \frac{1}{6} \gamma^{2} ; \frac{13}{30} \gamma^{2}[$ and $\beta=-5 \gamma$. It is very significant to underline here that, this family of solutions given by Equation (43) through its condition of existence: $\gamma=-5 \beta ; \alpha \in] \frac{1}{6} \gamma^{2} ; \frac{13}{30} \gamma^{2}[$, opens the way to other new varieties of fifth-order KdV equations [5] including her will be solution.

\subsubsection{Second Family of Solutions: Case: $a=0 ; b \neq 0$}

Under this condition, Equations (21), (23) and (25) are verified. However, Equation (26) gives

$$
v=\alpha c^{2}+\eta^{2} \gamma c+\eta^{4}
$$

while Equation (20) gives, successively

$$
b^{2}=3 c^{2}+\frac{\left(12 \eta^{2} \beta+24 \eta^{2} \gamma\right) c+360 \eta^{4}}{\alpha}
$$

and

$$
b= \pm \sqrt{3 c^{2}+\frac{\left(12 \eta^{2} \beta+24 \eta^{2} \gamma\right) c+360 \eta^{4}}{\alpha}}
$$

with $\alpha \neq 0$. Next, Equations (22) and (24) become respectively

$$
13 \eta \alpha c^{2}-3 \eta \alpha b^{2}+\left(28 \eta^{3} \beta+76 \eta^{3} \gamma\right) c+840 \eta^{5}=0
$$

and

$$
-8 \eta \alpha c^{2}+\eta \alpha b^{2}-\left(6 \eta^{3} \beta+29 \eta^{3} \gamma\right) c+2 \eta v-182 \eta^{5}=0
$$

On the one hand, by taking into account of Equation (45) in the Equation (47), and on the other hand, the insertion of Equations (44) and (45) in Equation (48) lead to the same quadratic equation in $c$ which is written

$$
r c^{2}+p c+q=0
$$


with $r=\alpha, p=\eta^{2} \gamma-2 \eta^{2} \beta$ and $q=-60 \eta^{4}$. Equation (49) has as discriminant

$$
\Delta=\eta^{4}(\gamma-2 \beta)^{2}+240 \eta^{4} \alpha .
$$

Looking at the architecture of Equation (50), a discussion according to the constraints linking $\gamma$ and $\beta$ becomes necessary. So, this discussion concerns the cases $\gamma=2 \beta ; \alpha \succ 0$ and the case $\gamma \neq 2 \beta ; \alpha \geq-\frac{(\gamma-2 \beta)^{2}}{240}$. From this investigation, the second family of solutions gives rise to two subfamilies.

1) First subfamily of the second family of solutions: case: $\gamma=2 \beta ; \alpha \succ 0$

Due to the fact that $\gamma=2 \beta ; \alpha \succ 0$, the discriminant becomes $\Delta=240 \eta^{4} \alpha \succ 0$ because $c \neq 0$. Therefore, Equation (49) admits as solution

$$
c= \pm \frac{2 \eta^{2}}{\alpha} \sqrt{15 \alpha}
$$

Equation (51) shows that, for a given value of $\alpha$, the coefficient $c$ is a parabolic function of the width at half height of each component of Equation (18). Thenceforward, the first subfamily of the second family of solutions is written considering Equation (46) under the form

$$
\begin{aligned}
\psi(\xi)= & \pm \sqrt{3 c^{2}+\frac{\left(12 \eta^{2} \beta+24 \eta^{2} \gamma\right) c+360 \eta^{4}}{\alpha}} J_{1,0}(\eta \xi) J_{1,1}(\eta \xi) \\
& \pm \frac{2 \eta^{2}}{\alpha} \sqrt{15 \alpha} J_{2,2}(\eta \xi)
\end{aligned}
$$

where $c$ is given by Equation (51). This solution presages interactions between hybrid and dark components of Equation (18). Which, in view of the constitution of the hybrid component of Equation (18), could generate bright, bright-dark, dark-bright, dark type structures, and so on, provided that $\alpha=2 \beta ; \alpha \succ 0$.

2) Second subfamily of the second family of solutions: case:

$\gamma \neq 2 \beta ; \alpha \geq-\frac{(\gamma-2 \beta)^{2}}{240}$

Under these conditions, the discriminant is that given by Equation (50). So, for $\Delta \geq 0$, the expression of $c$ solution of Equation (49) that we are looking for is in the form

$$
c=\frac{-p \pm \sqrt{\Delta}}{2 r}=\frac{2 \eta^{2} \beta-\eta^{2} \gamma \pm \sqrt{(\gamma-2 \beta)^{2}+240 \alpha}}{2 \alpha}
$$

Therefore, the second subfamily of the second family of solutions is written considering Equation (46) as being

$$
\begin{aligned}
\psi(\xi)= & \pm \sqrt{3 c^{2}+\frac{\left(12 \eta^{2} \beta+24 \eta^{2} \gamma\right) c+360 \eta^{4}}{\alpha}} J_{1,0}(\eta \xi) J_{1,1}(\eta \xi) \\
& +\frac{2 \eta^{2} \beta-\eta^{2} \gamma \pm \sqrt{(\gamma-2 \beta)^{2}+240 \alpha}}{2 \alpha} J_{2,2}(\eta \xi),
\end{aligned}
$$

where $c$ is given by Equation (53). This solution presages interactions between 
hybrid and dark components of Equation (18). Which, in view of the constitution of the hybrid component of Equation (18), could generate bright, bright-dark, dark-bright, dark type structures, and so on, provided that $\alpha=2 \beta ; \alpha \geq-\frac{(\gamma-2 \beta)^{2}}{240}$. We cannot forget to point out, in the case of this second family of solutions of Equation (1) that:

- the first subfamily given by Equation (52) through its condition of existence: $\gamma=2 \beta ; \alpha \succ 0$, opens the way to other new varieties of fifth-order KdV equations [5] including her will be solution;

- the second subfamily given by Equation (54) through its condition of existence: $\gamma \neq 2 \beta ; \alpha \geq-\frac{(\gamma-2 \beta)^{2}}{240}$ and $\alpha \neq 0$, opens the way to other new varieties of fifth-order KdV equations [5] including her will be solution. Now, One looks what is about the third family of solutions of the Equation (1).

\subsubsection{Third Family of Solutions: Case: $b=0 ; a \neq 0$}

For $b=0$ and $a \neq 0$, all the equations from (20) to (26) are verified for any nonzero values of the real coefficients $a ; c$ and those of the parameters $\alpha ; \beta ; \gamma ; \eta$ and $v$. According to the implementation of the BDKm as explained in Section 2, the third family of solutions can be approximated by the following expression

$$
\psi(\xi)=a J_{1,0}(\eta \xi)+c J_{2,2}(\eta \xi)
$$

with $a \in \mathfrak{R}^{*}$ and $c \in \mathfrak{R}^{*}$. Equation (55) presages interactions between bright and hybrid components of Equation (18). Which, in view of the constitution of the hybrid component of Equation (18), could generate bright, bright-dark, dark-bright, dark type structures, and so on, for nonzero values of $a ; c$ and those of the parameters $\alpha, \beta, \gamma, \eta$ and $v$. To fix the a priori ideas, a study of the numerical stability of the obtained solutions is necessary.

\subsubsection{Fourth Family of Solutions: Case: $a=0 ; b=0$}

By considering this condition, Equations (20) to (26) are verified. However, according to the BDK method, it is important in this case to examine what information can we derive from the equations of the second range (terms in $\left.J_{v, 1}(\alpha \xi), v \in\{2 ; 3 ; 4 ; 5 ; 6 ; 7\}\right)$. In this context, Equations (28), (30) and (32) are checked while, Equations (27), (29) and (31) are reduced to, respectively

$$
\begin{aligned}
& \alpha c^{2}+\left(6 \eta^{2} \beta+12 \eta^{2} \gamma\right) c+360 \eta^{4}=0, \\
& \alpha c^{2}+\left(2 \eta^{2} \beta+8 \eta^{2} \gamma\right) c+120 \eta^{4}=0
\end{aligned}
$$

and

$$
\alpha c^{2}+4 \eta^{2} \gamma c+16 \eta^{4}-v=0
$$

Equation (56) is a quadratic equation of the unknown coefficient $c$ and the discriminant of which is given by 


$$
\Delta=36 \eta^{4}\left[(\beta+2 \gamma)^{2}-40 \alpha\right] .
$$

From the architecture of Equation (59), the discussions according to the constraints between the parameters $\gamma$ and $\beta$ is necessary and thus offering two subfamilies of solutions for this fourth family, in particular, the subfamily characterized by $\beta=-2 \gamma ; \alpha \prec 0$ and the subfamily characterized by $\beta \neq-2 \gamma ; \alpha \leq \frac{(\beta+2 \gamma)^{2}}{40}$.

1) First subfamily of the fourth family of solutions: case: $\beta=-2 \gamma ; \alpha \prec 0$

Under the condition $\beta=-2 \gamma ; \alpha \prec 0$, Equation (59) leads to $\Delta=-1440 \eta^{4} \alpha \succ 0$ and then, Equation (56) has as solution

$$
c= \pm \frac{\sqrt{\Delta}}{2 \alpha}= \pm 6 \eta^{2} \frac{\sqrt{-10 \alpha}}{\alpha} \text {. }
$$

The insertion of Equation (60) in Equations (57) and (58), and, taking into account the constraint $\beta=-2 \gamma$ give successively

$$
\alpha=-\frac{1}{10} \gamma^{2}
$$

and

$$
v= \pm 240 \eta^{4} \frac{|\gamma|}{\gamma^{2}}-360 \eta^{4}
$$

So, we get this first subfamily of the fourth family of solutions of Equation (1) considering Equation (61) in the form

$$
\psi(\xi)= \pm 60 \eta^{2} \frac{|\gamma|}{\gamma^{2}} J_{2,2}(\eta \xi)
$$

Equation (63) shows that the fKdV equation has dark solitary wave solutions with a amplitude which is a parabolic function of the width at half height of the soliton for a given value of $\gamma \neq 0$.

2) Second subfamily of the fourth family of solutions: case:

$\beta \neq-2 \gamma ; \alpha \leq \frac{(\beta+2 \gamma)^{2}}{40}$

For $\beta \neq-2 \gamma ; \alpha \leq \frac{(\beta+2 \gamma)^{2}}{40}$ and $\Delta \geq 0$, the solution of Equation (56) is written

$$
c=\frac{-\left(3 \eta^{2} \beta+6 \eta^{2} \gamma\right) \pm 3 \eta \sqrt{(\beta+2 \gamma)^{2}-40 \alpha}}{\alpha} .
$$

Then, Equation (58) gives

$$
v=\alpha c^{2}+4 \eta^{2} \gamma c+16 \eta^{4}
$$

where $c$ is given by Equation (64) and should verify Equation (57). Thus, the second subfamily of the second family of solutions of Equation (1) has as ex- 
pression

$$
\psi(\xi)=\frac{-\left(3 \eta^{2} \beta+6 \eta^{2} \gamma\right) \pm 3 \eta \sqrt{(\beta+2 \gamma)^{2}-40 \alpha}}{\alpha} J_{2,2}(\eta \xi),
$$

where $\alpha \neq 0$. As in the case of Equation (63), the result given by Equation (66) also indicates that Equation (1) admits dark type solitons as solutions.

It should be said here that the solutions given by Equations (63) and (66) are exact solutions of Equation (1) of which one of the classes had been proposed in [12] through the tanh method. We can retain here that, for certain solutions constructed in this subsection by using the discriminant $\Delta$, only the cases $\Delta \geq 0$ are interesting because complex solutions are not part of the aims of this work.

\subsection{Numerical Simulations}

This section is dedicated to the numerical simulations aiming to make observable, reliable and applicable the obtained solutions. They (numerical simulations) also aim to confirm the theoretical predictions on the hybrid characters of the new prototypes of the obtained solitary waves within the framework of this work. To implement all of this, and knowing that the boundary conditions of the profiles of the solutions constructed in this manuscript are not identical at the two borders. We have used the MATLAB toolbox pdepe [45] which solves initial-boundary value problems for parabolic-elliptic PDEs in 1-D, with zero flux boundary conditions. We have also used spatially extended grids in others to minimize boundary reflections that could induce spurious effects. It is also necessary to point out here that, these boundary conditions are appropriate to the profiles of the solutions studied in this work, instead of the periodic boundary conditions which require that when a wave passes from one end (of the computational spatial grid) to the other which is opposite to it, it should keep the same properties.

We cannot continue this study without providing the reader with elements useful for a good understanding of the different spatiotemporal evolution curves obtained. For example, to obtain Figure 1(a), we have set $a=0.0257 ; b=-0.00192 ; c=0.0179 ; \alpha=0.245 ; \beta=6$ to infer $\gamma=-1.2 ; \nu=-0.000049492 ; \eta=0.0363$ of Equations (36), (40) and (41) respectively. In this context, discussions become necessary.

\section{Discussions}

From all that has been formulated in the previous lines, two poles clearly emerge and on which our discussions will focus. The first pole will be on the analytical results, while, the second pole will revolve around results revealed by numerical simulations. Thus:

${ }^{*}$ Speaking of analytical results, we can see that the existence conditions of the solutions given by Equations (43); (52) and (54), respectively, offer possibilities 


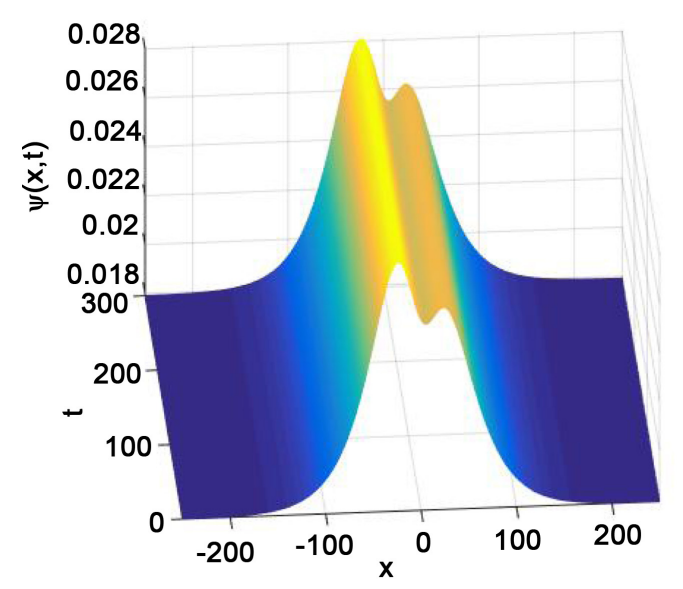

(a)

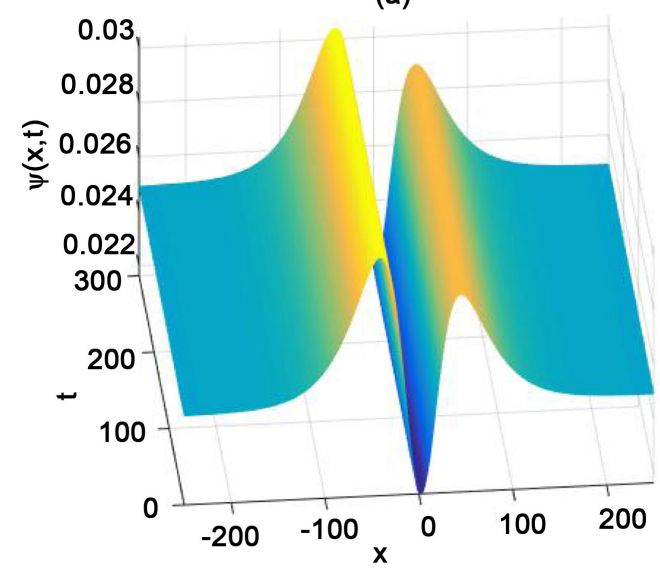

(c)
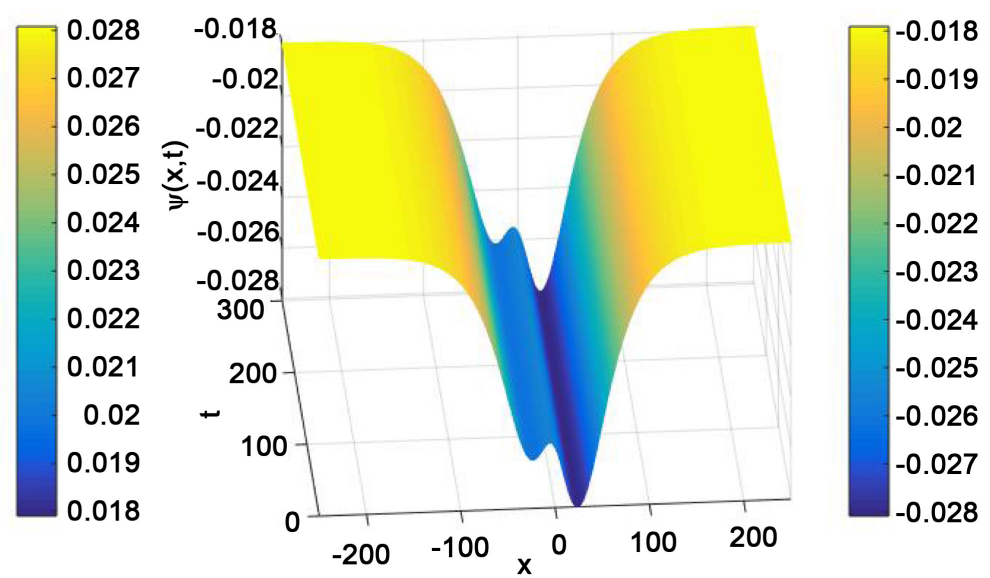

(b)
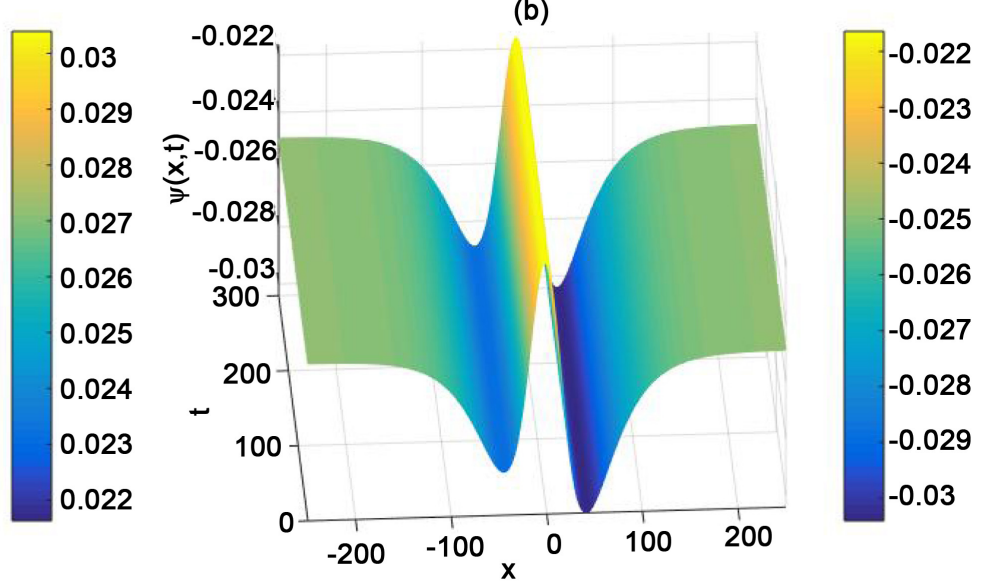

(d)

Figure 1. Robust dynamics for the hybrid solitary waves given by Equation (43). (a): $a=0.0257 ; b=-0.00192 ; c=0.0179$; $\alpha=0.245 ; \beta=6 ; \quad v=-0.000049492 ; \eta=0.0363 ; \gamma=-1.2$. (b): $a=-0.0257 ; b=-0.00192 ; c=-0.0179 ; \alpha=0.245 ;$ $\beta=6 ; \quad v=-0.000049492 ; \eta=0.0363 ; \gamma=-1.2$. (c): $a=0.0217 ; b=-0.00192 ; c=0.0249 ; \alpha=0.256 ; \beta=6 ;$ $v=-0.000037911 ; \eta=0.0344 ; \gamma=-1.2$. (d): $a=-0.0217 ; b=-0.00192 ; c=-0.0249 ; \alpha=0.256 ; \beta=-6 ;$ $v=0.00040707 ; \eta=0.0344 ; \gamma=1.2$.

to develop varieties of the fifth-order $\mathrm{KdV}$ equations by simply modifying [7] the real values of the parameters $\alpha, \beta$ and $\gamma$. Note here that all these varieties obtained must admit the ansatz given by Equation (18) as an approximate solution. To be a little clearer, one of the well-known fifth-order KdV equations is that of Lax, and which is characterized by:

$$
\beta=2 \gamma ; \alpha=(3 / 10) \gamma^{2} \text {. }
$$

For example, if $\gamma=10$, we obtain $\beta=20$ and $\alpha=30$, successively. Thus, Equation (1) becomes:

$$
\omega_{t}+30 \omega^{2} \omega_{x}+20 \omega_{x} \omega_{x x}+10 \omega \omega_{x x x}+\omega_{x x x x}=0
$$

Equation (68) is indeed the Lax equation which is one of the varieties of equations which derive from Equation (1) with values of parameters $\alpha, \beta$ and $\gamma$ given above. If we go back to our results:

- the solution given by Equation (43) has the existence condition 
$\beta=-5 \gamma ; \alpha \in] \frac{1}{6} \gamma^{2} ; \frac{13}{30} \gamma^{2}[$. For $\gamma=20$, we have $\beta=-100$ and $\alpha=100$.

These values taken by the coefficients $\gamma, \beta$ and $\alpha$ give rise to another equation which derives from Equation (1) and which is written:

$$
\omega_{t}+100 \omega^{2} \omega_{x}-100 \omega_{x} \omega_{x x}+20 \omega \omega_{x x x}+\omega_{x x x x x}=0
$$

and characterized by: $\beta=-5 \gamma ; \alpha=\frac{1}{4} \alpha^{2}$ and whose Equation (18) is an approximate solution.

- next, the solution given by Equation (52) has for existence condition $\beta=\frac{1}{2} \gamma ; \alpha \succ 0$. Let us take $\alpha$ in the form: $\alpha=\frac{1}{6} \gamma^{2}$. So, for $\gamma=30$, we have $\beta=15 ; \alpha=150$. So we get another form of Equation (1) as

$$
\omega_{t}+150 \omega^{2} \omega_{x}+15 \omega_{x} \omega_{x x}+30 \omega \omega_{x x x}+\omega_{x x x x x}=0
$$

whose solution given by Equation (18) is an approximate solution.

- eventually, a similar reasoning can also be carried out with the existence condition $\gamma \neq 2 \beta ; \alpha \geq-\frac{(\gamma-2 \beta)^{2}}{240}$ of the solutions given by Equation (54), in order to obtain the corresponding fifth-order KdV equations. It is necessary to point out here that the five well-known fifth order $\mathrm{KdV}$ equations in the literature can be tidy in this latter case.

It should be point out here that, Equations (69) and (70) are two new forms [7] among so many other fifth-order $\mathrm{KdV}$ equations that we have just revealed through this manuscript and which are characterized by $\beta=-5 \gamma ; \alpha \in] \frac{1}{6} \gamma^{2} ; \frac{13}{30} \gamma^{2}[$ and $\gamma=2 \beta ; \alpha \succ 0$, respectively, and whose Equations (43) and (52) are respective approximate solutions. From these existence conditions, we can propose new varieties of fifth-order $\mathrm{KdV}$ equations by simply altering the values of the different parameters $\alpha ; \beta$ and $\gamma$.

${ }^{\star}$ As for the numerical results, it appears by browsing from Figures 1-5 that:

- Figure 1 displays a set of four hybrid structures that we think are generated by balanced interactions between the three constituent solitons of the soliton package [23] [27] [28] [29] given by Equation (18). Figure 1(a) and Figure 1 (c) are unfinished intermediate forms of a double-bright soliton while, Figure 1(b) and Figure 1(d) are unfinished intermediate forms of a double-dark soliton respectively, obtained by simply altering the values of the coefficients $a, b$ and $c$.

- Figure 2 shows the dominant interactions of each of the terms making up Equation (18). We can observe this by noting that Figure 2(a) translates the domination of the bright soliton structure (first term of Equation (18)) while, Figure 2(b) and Figure 2(c) translate the domination of the hybrid soliton structure (second term of Equation (18)). Figure 2(d) translates the domination of the dark-soliton structure (third term of Equation (18)).

- Figure 3 presents a set of four profiles which reveal through Figure 3(a) and 


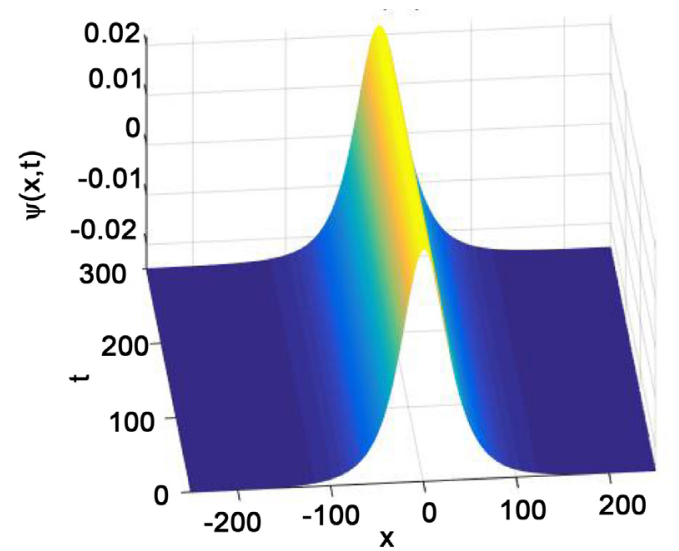

(a)

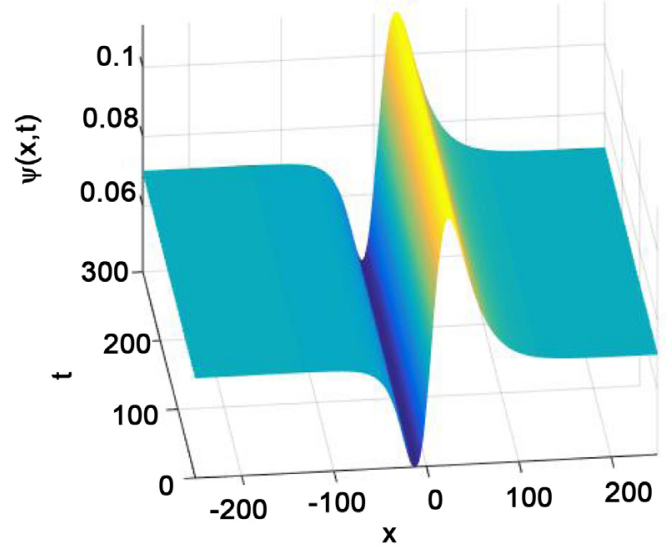

(c)

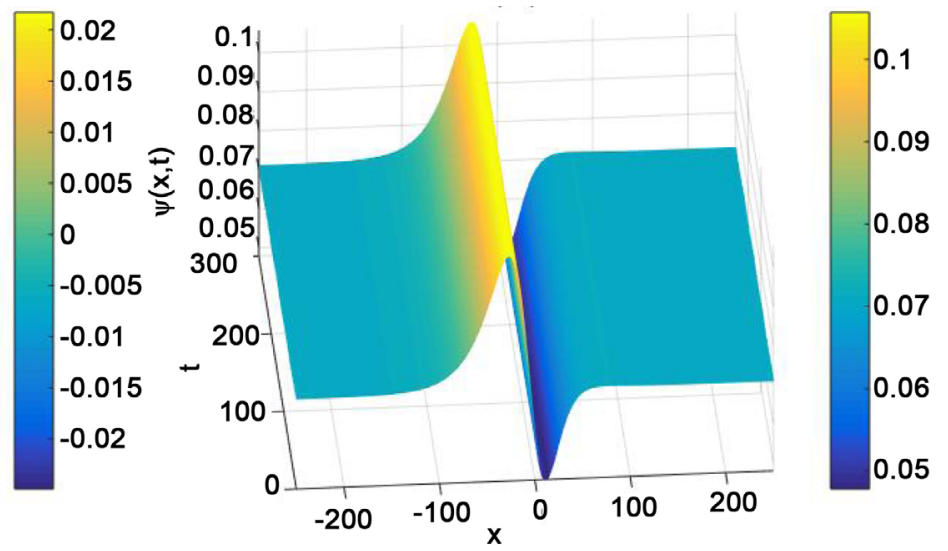

(b)
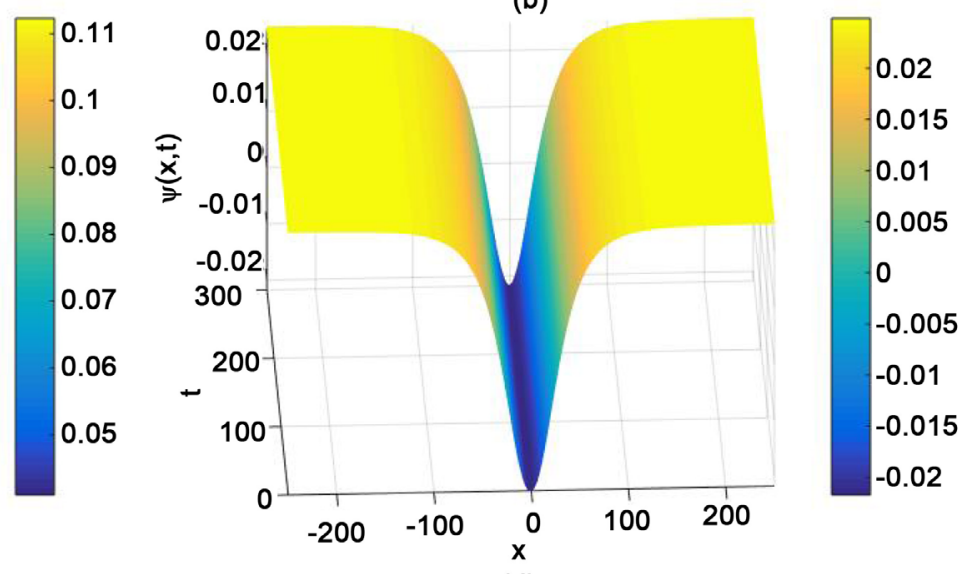

(d)

Figure 2. Stable dynamics of the bright-dark solitary waves given by Equation (43). (a): $a=0.0217 ; b=0.00192 ; c=-0.0179$; $\alpha=0.256 ; \beta=-6 ; v=0.000040707 ; \eta=0.0344 ; \gamma=1.2$. (b): $a=0.06 ; b=-0.0501 ; c=0.071 ; \alpha=0.07 ; \beta=-3 ;$ $v=-0.00017923 ; \eta=0.043 ; \gamma=0.6$. (c): $a=0.06 ; b=0.0657 ; c=0.07 ; \alpha=0.076 ; \beta=-3 ; v=-0.00020953 ;$ $\eta=0.0456 ; \gamma=0.6$. (d): $a=-0.0217 ; b=0.00192 ; c=-0.0249 ; \alpha=0.256 ; \beta=-6 ; \quad v=-0.000040707 ; \eta=0.0344 ;$ $\gamma=1.2$.

Figure 3(b) a dominance of hybrid structures with strong bright-soliton tendencies and given by Equation (52), while, Figure 3(c) and Figure 3(d) reveal a dominance of hybrid structures with strong dark-soliton tendencies and given by Equation (54). All these structures result from the interactions between the second term (hybrid soliton) and the third term (dark soliton) of Equation (18) in the absence of the first term (bright soliton).

- Figure 4 reveals four profiles which present improved and balanced forms of the structures obtained in Figure 1. Figure 4(a) is an intermediate form of a double-bright soliton with balanced vertices while Figure $4(\mathrm{~b})$ is an intermediate form of a double-dark soliton with balanced bottoms. On the other hand, Figure 4(c) presents an intermediate form of the double-bright soliton very evolved towards a bright-soliton with flat peak, then, Figure 4(d) is an intermediate form very evolved towards a dark-soliton form with flat bottom. One also has to point out here that all these figures are symmetrical with respect to a plane of equation $x=0$ and that these properties of symmetry 


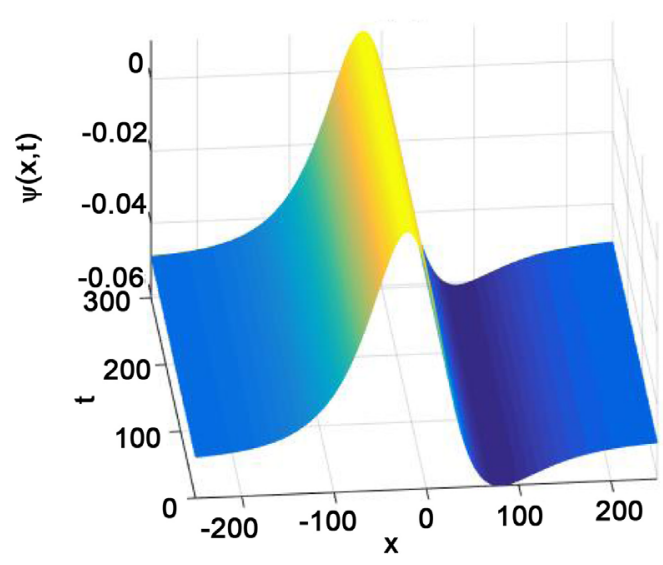

(a)

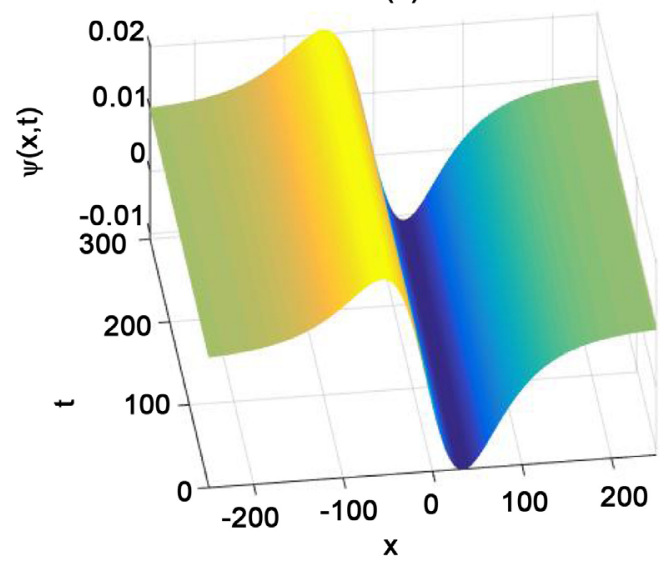

(c)
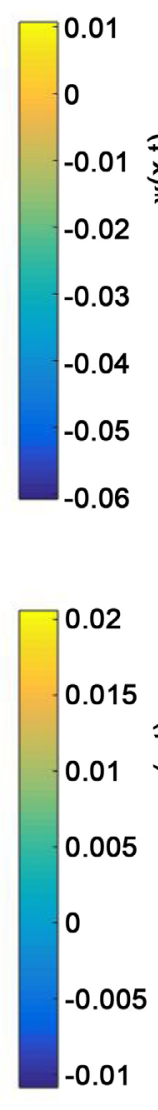

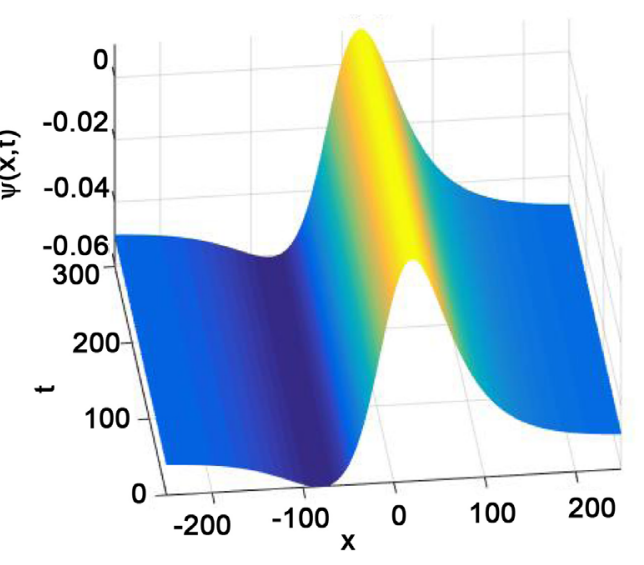

(b)

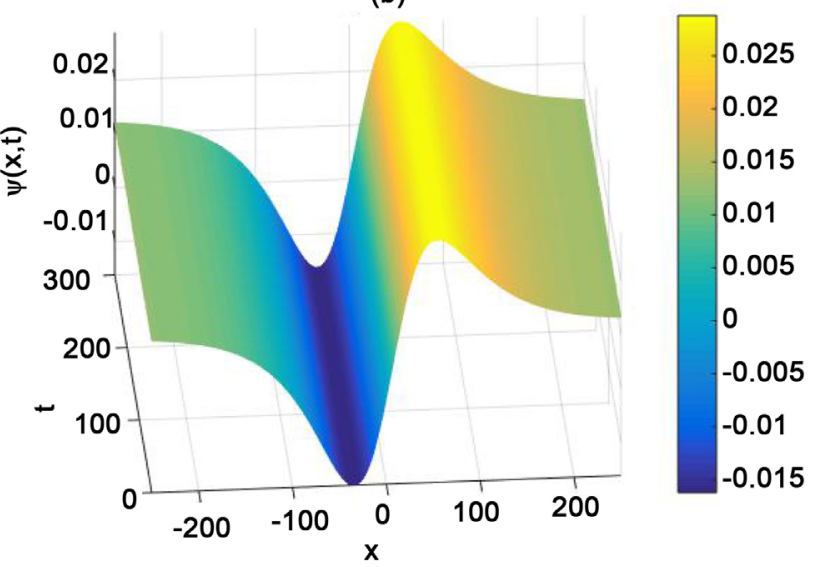

(d)

Figure 3. Stable spatiotemporal evolution of the hybrid bright-dark solitary waves given by Equation (52) and (54). (a): $a=0$; $b=-0.051 ; c=-0.05 ; \eta=0.02 ; \beta=3 ; \alpha=0.7231 ; \gamma=6 ; v=0.0017 ;(\mathrm{b}): a=0 ; b=0.051 ; c=-0.05 ; \eta=0.02 ;$ $\beta=3 ; \alpha=0.7231 ; \gamma=6 ; v=0.0017 ;(\mathrm{c}): a=0 ; b=-0.0299 ; c=0.0097 ; \eta=0.02 ; \beta=-0.09 ; \alpha=0.0997 ; \gamma=0.08 ;$ $v=0.0000098955 ;(\mathrm{d}): a=0 ; b=0.0432 ; c=0.0126 ; \eta=0.02 ; \beta=-0.09 ; \alpha=0.0718 ; \gamma=0.8 ; \nu=0.000015596$.

could be of capital importance in the understanding and the explanation of certain phenomena which occur in systems whose dynamics are described by Equation (1).

- Finally, Figure 5 completes the advanced forms presented in Figure 4. This is how Figure 5(a) shows a bright soliton structure with a flat peak and Figure 5 (b) shows a dark-soliton structure with a flat bottom. Figure 4 and Figure 5 translate the interactions between the first term (bright soliton) and the third term (dark soliton) of Equation (18) in the absence of the second term (hybrid soliton).

In summary, we find that, the analytical solutions obtained and of which we foresee hybrid characters have given rise to new varieties of fifth-order KdV equations while numerical simulations have confirmed the hybrid characters of most of the structures revealed starting from Figures 1-5. These solutions may find their applications in fluid mechanics, quantum mechanics, nonlinear optics, and so on. Abdul-Majid Wazwaz in [8] had used the simplified Hirota method to derive single, singular and multiple solitons with the auxiliary function, of 

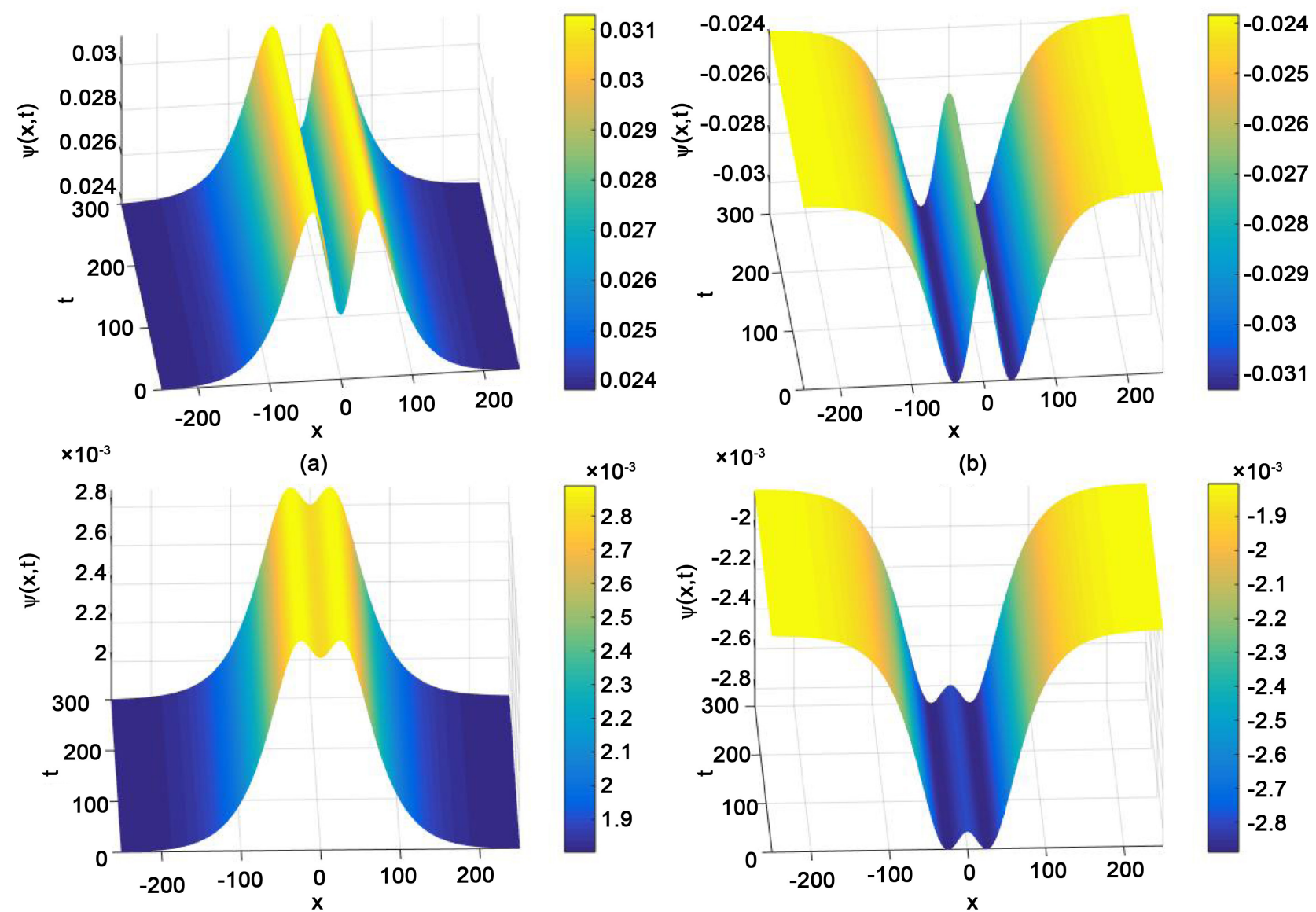

(c)

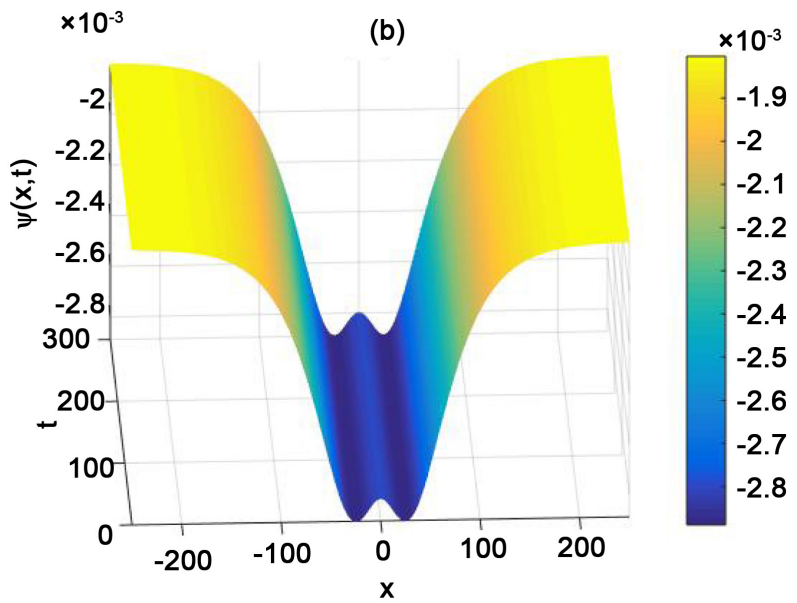

(d)

Figure 4. Robust dynamics of the multi-form solitary waves given by Equation (55). (a): $a=0.0267 ; b=0 ; c=0.0238$; $v=0.0013 ; \gamma=0.9 ; \beta=0.6 ; \eta=0.03 ; \alpha=1 .(\mathrm{b}): a=-0.0267 ; b=0 ; c=-0.0238 ; v=0.0013 ; \gamma=0.9 ; \beta=0.6 ;$ $\eta=0.03 ; \quad \alpha=1$. (c): $a=0.0028 ; b=0 ; c=0.0018 ; v=0.01 ; \gamma=0.9 ; \beta=0.6 ; \eta=0.03 ; \alpha=1 .(\mathrm{d}): a=-0.0028$; $b=0 ; c=-0.0018 ; v=0.01 ; \gamma=0.9 ; \beta=0.6 ; \eta=0.03 ; \alpha=1$.

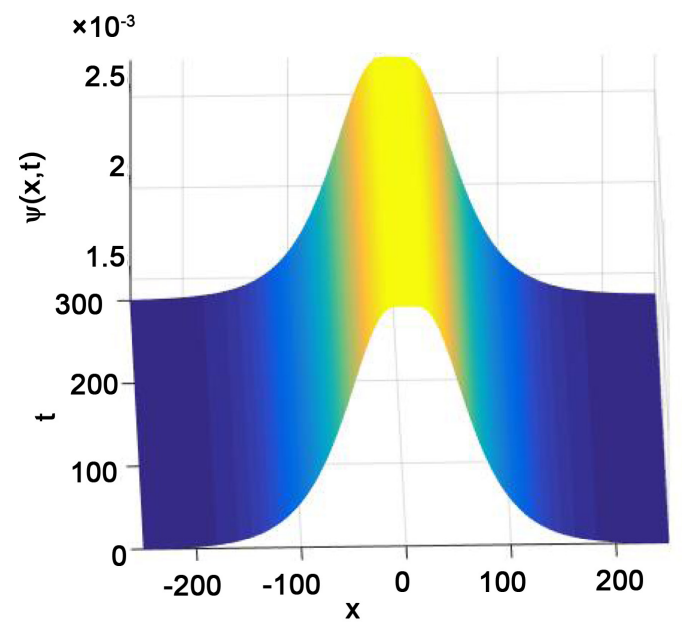

(a)

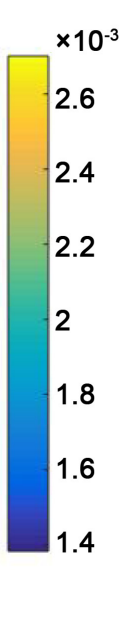

Figure 5. Stable spatiotemporal evolution of bright and dark solitons with a flat top and a flat bottom, respectively, given by Equation (55). (a): $a=0.0027 ; b=0 ; c=0.00138 ; \quad v=0.013 ; \gamma=0.9 ; \beta=0.6 ; \eta=0.03 ; \alpha=1 .(\mathrm{b}): a=-0.0027 ; b=0$; $c=-0.00138 ; \quad v=0.013 ; \gamma=0.9 ; \beta=0.6 ; \eta=0.03 ; \alpha=1$. 
Equation (1). He had also derived periodic solutions and compacton solutions of Equation (1) in [13]. Again, Abdul-Majid Wazwaz had used in [12] the sine-cosine and the tanh methods to successively derive exact periodic and soliton solutions of Equation (1). Bell shape multi-solitons, dark solitons and dark periodic cusp solutions of Equation (1) were found in [10] [11] while, exponential function solutions were revealed in [9]. It becomes easy to realize that, all these proposed solutions of Equation (1) including solutions found in [19] [25], are almost entirely different from those obtained in this manuscript.

\section{Conclusion}

In the final analysis, we can say with enthusiasm that, the application of the $\mathrm{BDKm}$ extended to iB-functions, to the standard form of the fifth-order KdV equations, has enabled to reveal new prototypes of solitary waves of higher order, which are for some, approximate hybrid (or multi-form) solutions, and for others, exact solutions of this equation. This method is very suitable for the construction of solitary wave solutions of certain types of equations in wave mechanics and which have terms of dispersion and terms of nonlinearity. The existence conditions of some of these solutions made it possible to derive from Equation (1) (and depending on the values of the parameters and depending on the values of the parameters $\alpha ; \beta$ and $\gamma$ ); some new varieties of the standard fifth-order KdV equations. Equation (18) is one of their multiple approximated solutions. Theoretical forecasts on the hybrid characters of the solutions obtained have been confirmed by intense numerical simulations as evidenced by the figures in Subsection 3.2. These figures also made it possible to understand that, when solitons are put together in the same package [23] [27] [28] [29], interactions occur between the different components of this package of solitons and what subsequently generates structures with hybrid characters. One estimates that, new varieties of equations derived from that of standard fifth-order KdV, including the new solutions proposed in this manuscript, will allow, during the propagations tests in the laboratories, to analyze, understand and explain some old or new phenomena which occur or which would occur in the systems whose dynamics are governed by Equation (1), in particular during the propagation of waves of low amplitudes and long wavelength on the surface of shallow waters [15] [16], during the transport of data through non-linear optical fibers and many other phenomena in quantum mechanics, in order to better secure our existence. One also hopes that, the properties of symmetry which certain obtained structures have, could be of capital importance in the understanding and the explanation of certain phenomena which occur in systems whose dynamics are described by Equation (1). It should also be pointed out that, the possibilities of formulating new varieties of fKdV-type equations offered by the results of this manuscript will help in the future, to devise new shallow fluid media with improved properties. However, the universe as a whole is being perpetually dynamic. We must further explore these models in order to obtain every day new 
information which is still hidden behind this equation for the benefit of all humanity and which will guarantee at the same time our survival.

\section{Acknowledgements}

The authors would like to thank both ministries of higher education of Cameroon and Gabon for their assistance through their respective research support programs. The authors' thanks go again to Keutcha Padji Aurélien whose assistance in reading this manuscript has contributed to improving the quality of the English used. Authors would also like to thank the reviewers whose relevance of their criticism has enabled us to more improve the quality of this work.

\section{Conflicts of Interest}

The authors declare no conflicts of interest regarding the publication of this paper.

\section{References}

[1] Das, A. and Popowicz, Z. (2005) A Nonlinearly Dispersive Fifth Order Integrable Equation and Its Hierarchy. Journal of Nonlinear Mathematical Physics, 12, 105-117. https://doi.org/10.2991/jnmp.2005.12.1.9

[2] Kudryashov, N.A. (2019) General Solution of Traveling Wave Reduction for the Kundu-Mukherjee-Naskar Model. Optik, 186, 22-27. https://doi.org/10.1016/j.ijleo.2019.04.072

[3] Nasreen, N., Seadawy, A.R. and Lu, D. (2019) Study of Modulation Instability Analysis and Optical Soliton Solutions of Higher-Order Dispersive Nonlinear Schrödinger Equation with Dual-Power Law Nonlinearity. Modern Physics Letters B, 33, Article ID: 1950309. https://doi.org/10.1142/S0217984919503093

[4] He, G.-L. and Geng, X.G. (2012) An Extension of the Modified Sawada-Kotera Equation and Conservation Laws. Chinese Physics B, 21, Article ID: 070205. https://doi.org/10.1088/1674-1056/21/7/070205

[5] Azad, H., Lubna, S., Sobia, A. and Sohail, N. (2020) Mathematical Model for Blood Flow through the Stenosed Channel. Physica Scripta, 95, Article ID: 025206. https://doi.org/10.1088/1402-4896/ab43ff

[6] Russell, J.S. (1844) Report on Waves. Report of the Fourteenth Meeting of the British Association for the Advancement of Science.

[7] Wazwaz, A.M. (2009) Partial Differential Equations and Solitary Waves Theory. Higher Education Press, Beijing and Springer-Verlag, Berlin Heidelberg, 562. https://doi.org/10.1007/978-3-642-00251-9_12

[8] Wazwaz, A.M. (2016) A Fifth-Order Korteweg-de Vries Equation for Shallow Waterwith Surface Tension: Multiple Soliton Solutions. Acta Physica Polonica A, 130, 679-682. https://doi.org/10.12693/APhysPolA.130.679

[9] Seadawy, A.R., Nuruddeen, R.I., Aboodh, K.S. and Zakariya, Y.F. (2020) On the Exponential Solutions to Three Extracts from Extended Fifth-Order KdV Equation. Journal of King Saud University-Science, 32, 765-769. https://doi.org/10.1016/j.jksus.2019.01.007

[10] Ashrafuzzaman, K.M. and Ali, A.M. (2015) Exact and Solitary Wave Solutions to the Generalized Fifth-Order KdV Equation by Using the Modified Simple Equation 
Method. Applied and Computational Mathematics, 4, 122-129. https://doi.org/10.11648/j.acm.20150403.14

[11] Xiang, C.H. and Wang, H.L. (2019) Travelling Solitary Wave Solutions to Higher Order Korteweg-de Vries Equation. Open Journal of Applied Sciences, 9, 354-360. https://doi.org/10.4236/ojapps.2019.95029

[12] Wazwaz, A.M. (2006) Solitons and Periodic Solutions for the Fifth-Order KdV Equation. Applied Mathematics Letters, 19, 1162-1167. https://doi.org/10.1016/j.aml.2005.07.014

[13] Wazwaz, A.M. (2005) Variants of the Two-Dimensional Boussinesq Equation with Compactons, Solitons, and Periodic Solutions. Computers \& Mathematics with Applications, 49, 295-301. https://doi.org/10.1016/j.camwa.2004.06.029

[14] Shivamoggi, B.K. (1988) Introduction to Nonlinear Fluid-Plasma Waves. Kluwer Academic Publishers, Dordrecht. https://doi.org/10.1007/978-94-009-2772-8

[15] Whitham, G.B. (1974) Linear and Nonlinear Waves, In: Pure and Applied Mathematics, Wiley/Interscience, New York, 431.

[16] Korteweg, D.J. and de Vries, G. (1895) On the Change of Form of Long Waves Advancing in a Rectangular Canal, and on a New Type of Long Stationary Waves. Philosophical Magazine Series 5, 39, 422-443. https://doi.org/10.1080/14786449508620739

[17] Debussche, A. and Printems, J. (1999) Numerical Simulation of the Stochastic Korteweg-de Vries Equation. Physica D: Nonlinear Phenomena, 134, 200-226. https://doi.org/10.1016/S0167-2789(99)00072-X

[18] Rabinovich, M.I. and Trubetskov, T.D. (1984) Introduction to Theory of Oscillations and Waves. Nauka, Moscow, 6.

[19] Ganji, D.D. and Abdollahzadeh, M. (2008) Exact Travelling Solutions for the Lax's Seventh-Order KdV Equation by Sech Method and Rational Exp-Function Method. Applied Mathematics Computation, 206, 438-444.

https://doi.org/10.1016/j.amc.2008.09.033

[20] Aljahdaly, N.H., Seadawy, A.R. and Albarakati, W.A. (2019) Applications of Dispersive Analytical Wave Solutions of Nonlinear Seventh Order Lax and Kaup-Kupershmidt Dynamical Wave Equations. Results in Physics, 14, Article ID: 102372. https://doi.org/10.1016/j.rinp.2019.102372

[21] Xiang, C.H. and Wang, H.L. (2020) New Exact Solutions for Benjamin-Bona-Mahony-Burgers Equation. Open Journal of Applied Sciences, 10, 543-550. https://doi.org/10.4236/ojapps.2020.108038

[22] Djeumen Tchaho, C.T. (2015) New Method of Construction of the Solitary Wave Solutions of Some Physical Nonlinear Partial Differential Equations. Doctorate/PhD Thesis, University of Yaounde I, Cameroon.

[23] Bogning, J.R. (2019) Mathematics for Nonlinear Physics: Solitary Waves in the Center of Resolutions of Dispersive Nonlinear Partial Differential Equations. Dorrance Publishing Co., Pittsburgh.

[24] Seadawy, A.R. and Lu, D. (2017) Bright and Dark Solitary Wave Soliton Solutions for the Generalized Higher Order Nonlinear Schrödinger Equation and Its Stability. Results in Physics, 7, 43-48. https://doi.org/10.1016/j.rinp.2016.11.038

[25] Seadawy, A.R., Lu, D. and Yu, C. (2017) Travelling Wave Solutions of the Generalized Nonlinear Fifth-Order KdV Water Wave Equations and Its Stability. Journal of Taibah University-Science, 11, 623-633.

https://doi.org/10.1016/j.jtusci.2016.06.002 
[26] Biswas, A., Milovic, D., Savescu, M., Mahmood, M.F., Khan, K.R. and Kohl, R. (2012) Optical Soliton Perturbation in Nanofibers with Improved Nonlinear Schrödinger Equation by Semi-Inverse Variational Principle. Journal of Nonlinear Optical Physics \& Materials, 21, Article ID: 1250054. https://doi.org/10.1142/S0218863512500543

[27] Bogning, J.R. (2019 Mathematics for Nonlinear Physics: The Implicit Bogning Functions and Applications. Lambert Academic Publishing, Saarbrücken.

[28] Bogning, J.R. (2020) Elements of Analytical Mechanics and Quantum Physics. Lambert Academic Publishing, Saarbrücken.

[29] Ngouo Tchinda, C. and Bogning, J.R. (2020) Solitary Waves and Property Management of Nonlinear Dispersive and Flattened Optical Fiber. American Journal of $O p$ tics and Photonics, 8, 27-32. https://doi.org/10.11648/j.ajop.20200801.13

[30] Bogning, J.R. (2018) Exact Solitary Wave Solutions of the (3+1)-Modified B-Type Kadomtsev-Petviashvili Family Equations. American Journal of Computational and Applied Mathematics, 8, 85-92.

[31] Djeumen Tchaho, C.T., Bogning, J.R. and Kofané, T.C. (2010) Construction of the Analytical Solitary Wave Solutions of Modified Kuramoto-Sivashinsky Equation by the Method of Identification of Coefficients of the Hyperbolic Functions. Far East Journal of Dynamical Systems, 14, 14-17.

[32] Bogning, J.R., Djeumen Tchaho, C.T. and Kofané, T.C. (2012) Construction of the Soliton Solutions of the Ginzburg-Landau Equations by the New Bogning-Djeumen Tchaho-Kofané Method. Physica Scripta, 85, 025013-025017. https://doi.org/10.1088/0031-8949/85/02/025013

[33] Djeumen Tchaho, C.T., Bogning, J.R. and Kofané, T.C. (2011) Multi-Soliton Solutions of the Modified Kuramoto-Sivashinsky Equation by the BDK Method. Far East Journal of Dynamical Systems, 15, 83-98.

[34] Djeumen Tchaho, C.T., Bogning, J.R. and Kofané, T.C. (2012) Modulated Soliton Solution of the Modified Kuramoto-Sivashinsky's Equation. American Journal of Computational and Applied Mathematics, 2, 218-224.

https://doi.org/10.5923/j.ajcam.20120205.03

[35] Bogning, J.R., Djeumen Tchaho, C.T. and Kofané, T.C. (2012) Generalization of the Bogning-Djeumen Tchaho-Kofané Method for the Construction of the Solitary Waves and the Survey of the Instabilities. Far East Journal of Dynamical Systems, 20, 101-111.

[36] Djeumen Tchaho, C.T., Omanda, H.M. and Belobo Belobo, D. (2018) Hybrid Solitary Waves for the Generalized Kuramoto-Sivashinsky Equation. European Physical Journal Plus, 133, 387-394. https://doi.org/10.1140/epjp/i2018-12218-4

[37] Djeumen Tchaho, C.T., Omanda, H.M., N’tchayi Mbourou, G., Bogning, J.R. and Koafné, T.C. (2019) Multi-Form Solitary Waves Solutions of the KdV-Burgers-Kuramoto Equation. Journal of Physics Communications, 3, Article ID: 105013. https://doi.org/10.1088/2399-6528/ab4bal

[38] Njikue, R., Bogning, J.R. and Kofané, T.C. (2018) Exact Bright and Dark Solitary Wave Solutions of the Generalized Higher-Order Nonlinear Schrödinger Equation Describing the Propagation of Ultra-Short Pulse in Optical Fibers. Journal of Physics Communications, 2, Article ID: 025030. https://doi.org/10.1088/2399-6528/aaaf3b

[39] Bogning, J.R., Djeumen Tchaho, C.T. and Omanda, H.M. (2016) Combined Solitary Wave Solutions in Higher-Order Effects Optical Fibers. British Journal of Mathematics \& Computer Science, 13, 1-12. https://doi.org/10.9734/BJMCS/2016/10620 
[40] Bogning, J.R., Fautso Kuiaté, G., Omanda, H.M. and Djeumen Tchaho, C.T. (2015) Combined Peakons and Multiple-Peak Solutions of the Camassa-Holm and Modified KdV Equations and Their Conditions of Obtention. Physics Journal, 3, 367-374.

[41] Bogning, J.R., Porsezian, K., Fautso Kuiaté, G. and Omanda, H.M. (2015) Gap Solitary Pulses Induced by the Modulational Instability and Discrete Effects in Array of Inhomogeneous Optical Fibers. Physics Journal, 1, 216-224.

[42] Tiague Takongmo, G. and Bogning, J.R. (2018) Construction of Solutions in the Shape (Pulse; Pulse) and (Kink; Kink) of a Set of Two Equations Modeled in a Nonlinear Inductive Electrical Line with Crosslink Capacitor. American Journal of Circuits, Systems and Signal Processing, 4, 28-35.

[43] Tiague Takongmo, G. and Bogning, J.R. (2018) Construction of Breather Soliton Solutions of a Modeled Equation in a Discrete Nonlinear Electrical Line and the Survey of Modulationnal Instability. Journal of Physics Communications, 2, Article ID: 115007. https://doi.org/10.1088/2399-6528/aaeaa1

[44] Tiague Takongmo, G. and Bogning, J.R. (2018) Coupled Soliton Solutions of Modeled Equations in a Noguchi Electrical Line with Crosslink Capacitor. Journal of Physics Communications, 2, Article ID: 105016. https://doi.org/10.1088/2399-6528/aae7e6

[45] Skeel, R.D. and Berzins, M. (1990) A Method for the Spatial Discretization of Parabolic Equations in One Space Variable. SIAM Journal on Scientific and Statistical Computing, 11, 1-32. https://doi.org/10.1137/0911001 